\title{
Testing the Reverse Monte Carlo Method Using Scattering Data from Amorphous $\mathrm{Ni}_{2} \mathrm{Zr}$
}

\author{
M. Bionducci, F. Buffa, G. Licheri, and G. Navarra \\ Dipartimento di Scienze Chimiche, Universitá di Cagliari, Via Ospedale, Cagliari, Italy
}

Z. Naturforsch. 47 a, 685-688 (1992); received February 3, 1992

The reverse Monte Carlo simulation has been tested by modelling the partial distribution functions of amorphous $\mathrm{Ni}_{2} \mathrm{Zr}$. Three runs of simulation involving experimental data from isotopic substitutions neutron scattering and X-ray anomalous scattering are presented.

\section{Introduction}

Many efforts have been made in the last years to obtain information about the short medium range structure of metallic glasses by neutron and X-ray scattering techniques which provide the total distribution function $g(r)$. Moreover, by using neutron scattering with isotopic substitutions or anomalous X-ray scattering, it is possible to perform, in some favourable cases, the separation of the three partial distribution functions (PDF) $g_{i j}(r)$ for a binary system. Unfortunately, this kind of information is only one-dimensional and is not sufficient to produce a three-dimensional model of the amorphous structure.

Computer simulation (Monte Carlo, Molecular Dynamics) should provide three-dimensional information; however, this requires suitable interatomic potentials that accurately reproduce the observed structural features, which is not a simple matter.

Recently, a new method has been suggested, the Reverse Monte Carlo method (RMC) [1] that provides a three-dimensional model of the structure able to fit the experimental $g(r)$, or any other structural function, without interatomic potentials as input.

RMC has successfully been applied on covalent compounds [2] and molten salts [3-5], while some difficulties have been reported in the case of high number density systems such as amorphous metal-metalloid alloys [6].

We are working on the structural properties of metal-metal amorphous alloys and we are interested

Reprint requests to Prof. G. Licheri, Dipartimento di Scienze Chimiche, Universitá di Cagliari, Via Ospedale, 72, I-09124 Cagliari, Italy. in applying RMC on these materials. Here an initial test on a $\mathrm{Ni}_{2} \mathrm{Zr}$ amorphous alloy for which suitable PDF are available $[7,8]$ is reported. In the following we give a summary of the RMC method and present the results obtained by using experimental data from both neutron (run 1, run 2) and X-ray scattering (run 3) as input information.

\section{Reverse Monte Carlo Method}

The RMC has been described in detail elsewhere [2, 9], thus only a brief summary is given here. In synthesis, RMC is a variation of the standard Metropolis Monte Carlo procedure in which the sequence of configurations is systematically compared with experimental data until convergence is reached.

The following steps elucidate the procedure:

i) An initial configuration of $N$ particles in a periodic cubic box of side length $L$ is created. The size of the box must be chosen such that the number density corresponds to the experimental one. The starting configuration may be a lattice, a random network or the result of an earlier simulation.

ii) Starting, simulated $g_{\mathrm{ij}}(r)$ functions are calculated and from them any function $F_{\mathrm{c}}(x)$ of interest can be constructed. $F_{\mathrm{c}}(x)$ is compared with the corresponding experimental function $F_{\mathrm{e}}(x)$, via a standard $\chi^{2}$-test

$$
\chi^{2}=\sum_{k}\left[F_{\mathrm{c}}\left(x_{k}\right)-F_{\mathrm{e}}\left(x_{k}\right)\right]^{2} / 2 \sigma_{k}^{2},
$$

where $\sigma_{k}$ is the estimated experimental error.

iii) A new configuration is generated by a random motion of a randomly chosen particle. This produces a new $F_{c}(x)$, and a $\chi^{2 \prime}$ can be calculated. 
iv) If $\chi^{2}>\chi^{2 \prime}$ the move is accepted, otherwise it is accepted with a probability $\exp \left(\chi^{2 \prime}-\chi^{2}\right)$. The procedure is repeated from (iii).

As the procedure is iterated, $\chi^{2}$ will decrease to an equilibrium value about which it will oscillate. At this point it is possible to start collecting a set of independent configurations consistent with the experimental data and calculate the average of $F_{\mathrm{c}}(x)$.

RMC is essentially a fitting method, but it presents the advantage that the set of simulated functions is self consistent and corresponds to a real distribution of particles. This makes it possible to draw structural information, such as bond angle distributions, not available directly from experiments.

\section{RMC Simulation on $\mathrm{Ni}_{2} \mathrm{Zr}$}

As first experimental reference, the three partial structure factors kindly supplied by S. Lefebvre were considered. They were obtained by neutron scattering measurements on rapidly quenched $\mathrm{Ni}_{2} \mathrm{Zr}$ samples with different isotopic compositions [7]. The partial structure factors have been Fourier transformed to obtain the $g_{i j}(r)$ and then the total distribution function $g(r)$ in the formalism of Faber-Ziman:

$$
\begin{aligned}
& g(r)=w_{\mathrm{Ni}-\mathrm{Ni}} g_{\mathrm{Ni}-\mathrm{Ni}}(r)+2 w_{\mathrm{Ni}-\mathrm{Zr}} g_{\mathrm{Ni}-\mathrm{Zr}}(r) \\
&+w_{\mathrm{Zr}-\mathrm{Zr}} g_{\mathrm{Zr}-\mathrm{Zr}}(r), \\
& w_{i j}=c_{i} c_{j} b_{i} b_{j} /\left(c_{i} b_{i}+c_{j} b_{j}\right)^{2},
\end{aligned}
$$

where $b_{i}, b_{j}$ are the coherent scattering lengths for ${ }^{\text {nat }} \mathrm{Ni}$ and ${ }^{\text {nat }} \mathrm{Zr}$ [10] and $c_{\mathrm{Ni}}, c_{\mathrm{Zr}}$ are the compositional coefficients.

In run 1 , we modelled the total distribution function, while in run 2 the three experimental PDF were directly used as input for the simulation.

In run 3, the input data consisted in the PDF obtained by X-ray anomalous scattering from an $\mathrm{Ni}_{2} \mathrm{Zr}$ sample prepared by mechanical alloying [8].

In all runs the starting configuration was a random box of 621 atoms in a cube of $L=21.07 \AA$. The simulation constraints were the exprimental number density and the closest distances that two atoms are allowed to approach: $r_{\mathrm{Ni}-\mathrm{Ni}}=2.18 \AA, r_{\mathrm{Ni}-\mathrm{Zr}}=2.29 \AA$, $r_{\mathrm{Zr}-\mathrm{Zr}}=2.7 \AA$. The experimental error $\sigma_{k}$ was kept constant. After convergence, ten independent configurations were collected to calculate average partial and total distribution functions. The acceptance ratio was about $1: 20$, slightly decreasing near convergence.

\section{Result and Discussion}

The results of the different simulation runs are presented in Figs. 1-4 and compared with the exprimental references.

In run 1, where only a total $g(r)$ has been used as input information, a very good reconstruction of the experimental $g(r)$ has been reached with a rapid convergence. However, the three calculated PDF which compose thre total $g(r)$ do not fit well the corresponding experimental ones. Evidently, the input experimental information is not sufficient to describe the details of the atomic organization. As can be seen in Fig. 4 (run 1), the simulation is particularly poor in the case of the $\mathrm{Zr}-\mathrm{Zr}$ PDF, where the distribution of the near nearest distances appears to be asymmetric and peaked very close to the hard sphere diameter. This is probably due to the combined effect of the low weight of the $\mathrm{Zr}-\mathrm{Zr}$ contribution and the high number density which hampers the mobility of the $\mathrm{Zr}$ atoms.

In run 2, the increasing of input information produces a clear improvement in the agreement between the experimental and simulated functions, as shown in Figs. 1-4. This result suggests that, when accurate scattering measurements covering an extended range of reciprocal space are available, the PDF represent a suitable input information for the RMC simulation.

In run 3, the aim is to test the effect of systematic errors on RMC. In fact, the sample used to perform $\mathrm{X}$-ray anomalous scattering measurements is affected by a residual $\mathrm{Zr}$ crystalline phase which introduces spurious contributions in the experimental data. As a consequence, the experimental PDF involving $\mathrm{Zr}$ atoms exhibit some distorsions with respect to those obtained by neutron scattering, apart the different resolution due to different truncation limits in performing Fourier transform.

A result of run 3 is especially interesting. That is, the capability of RMC in refusing to reproduce the details of experimental curves particularly affected by nonphysical behaviour. Looking at Figs. 3 and 4 (run 3), two observations can be easily made: i) the heighest of the two maxima in the range $4-6 \AA$ of the simulated $\mathrm{Zr}-\mathrm{Zr}$ PDF are inverted with respect to experimental curve, ii) the simulated $\mathrm{Ni}-\mathrm{Zr}$ PDF does not fit the region after the main peak. In both cases, the simulation results look like the correct behaviour shown in run 2. On the contrary, the $\mathrm{Ni}-\mathrm{Ni} \mathrm{PDF}$, which is not affected by distorsions, is well reproduced by the simulation. 
M. Bionducci et al. - Scattering Data from Amorphous $\mathrm{Ni}_{2} \mathrm{Zr}$

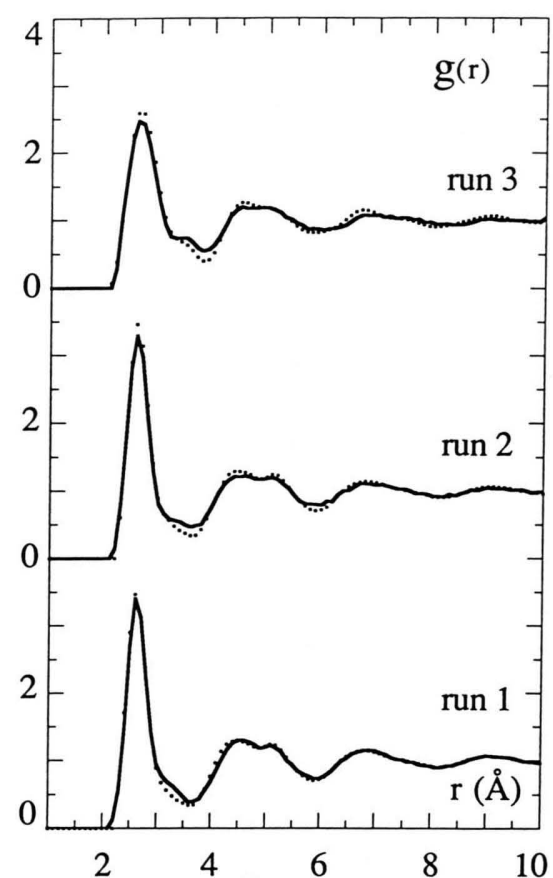

Fig. 1. Simulated (solid curves) and experimental (dotted curves) total distribution functions. The experimental curves come from [7] (run 1 and run 2) and from [8] (run 3).

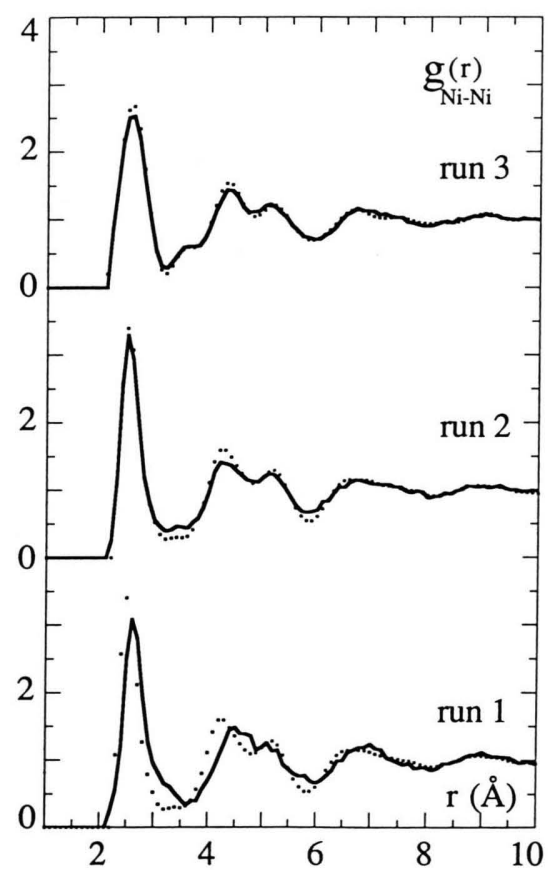

Fig. 2. Simulated (solid curves) and experimental (dotted curves) $\mathrm{Ni}-\mathrm{Ni}$ partial distribution functions. The expermental curves come from [7] (run 1 and run 2) and from [8] (run 3).

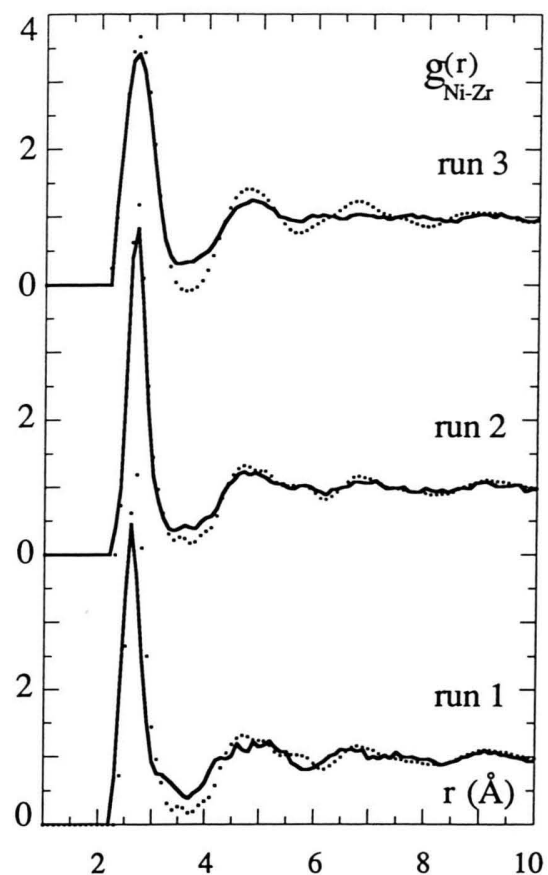

Fig. 3. Simulated (solid curves) and experimental (dotted curves) $\mathrm{Ni}-\mathrm{Zr}$ partial distribution functions. The experimental curves come from [7] (run 1 and run 2) and from [8] (run 3).

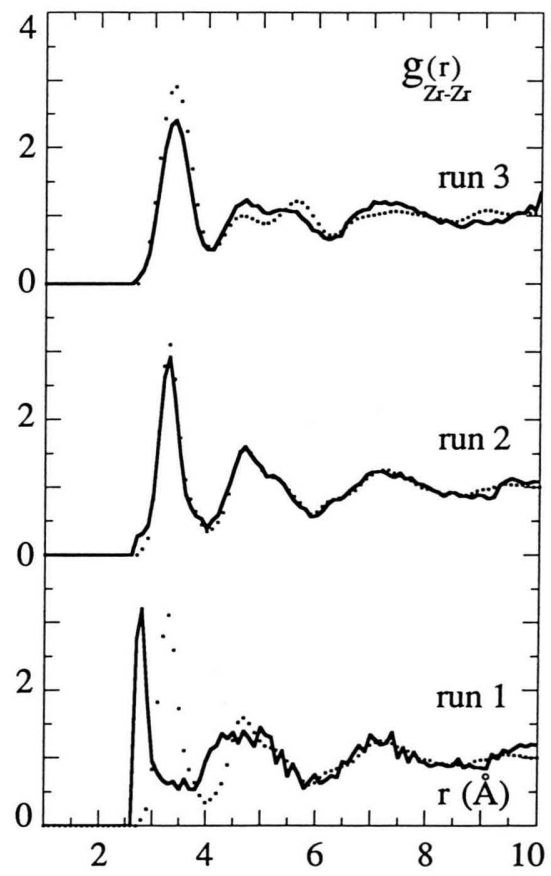

Fig. 4. Simulated (solid curves) and experimental (dotted curves) $\mathrm{Zr}-\mathrm{Zr}$ partial distribution functions. The expermental curves come from [7] (run 1 and run 2) and from [8] (run 3). 


\section{Conclusions}

If a suitable input information is available, $\mathrm{RMC}$ can produce good quality simulations of both total and partial distribution functions. This represents the first step by which structural properties of disordered materials may be investigated. Furthermore, RMC

1] R. L. McGreevy and L. Pusztai, Mol. Sim. 1, 359 (1988).

[2] D. A. Keen and R. L. McGreevy, Nature 344, 423 (1990).

[3] R. L. McGreevy, Inst. Phys. Conf. Ser. 101, 41 (1989).

[4] R. L. McGreevy, L. Pusztai, Proc. Roy. Soc. London A 430, 241 (1990).

[5] M. A. Howe and R. L. McGreevy, J. Phys.: Cond. Matter 3, 577 (1991).

[6] L. Pusztai, Z. Naturforsch. 46a, 69 (1991). seems to have the capability to reveal flows in experimental data.

\section{Acknowledgements}

This work has been supported by Consiglio Nazionale delle Ricerche (Italy).

[7] S. Lefebvre, A. Quivy, J. Bigot, Y. Calvayrac, and R. Bellissent, J. Phys. F Met. Phys. 15, 99 (1985).

[8] F. Buffa, A. Corrias, G., Licheri, G. Navarra, and D. Raoux, to be published.

[9] R. L. McGreevy, M. A. Howe, D. A. Keen, and K. N. Clausen, Inst. Phys. Conf. Ser. 107, 165 (1990).

[10] C. G. Windsor, Published Neutron Scattering, Tailor \& Francis Ltd., London 1981. 